\title{
The Highlighted Role of GAPDH and Nitric-Oxide Synthase Regulator Activity in Proton Beam Irradiated Melanoma BLM Cells
}

\author{
Mohammad-Mehdi Zadeh-Esmaeel', Shabnam Shahrokh², Mona Zamanian Azodi ${ }^{3}{ }^{*}$, Nayebali Ahmadi ${ }^{4}$ \\ 'Laser Application in Medical Sciences Research Center, Shahid Beheshti University of Medical Sciences, Tehran, Iran \\ ${ }^{2}$ Gastroenterology and Liver Diseases Research Center, Research Institute for Gastroenterology and Liver Diseases, Shahid \\ Beheshti University of Medical Sciences, Tehran, Iran \\ ${ }^{3}$ Proteomics Research Center, Shahid Beheshti University of Medical Sciences, Tehran, Iran \\ ${ }^{4}$ Proteomics Research Center, Faculty of Paramedical Sciences, Shahid Beheshti University of Medical Sciences, Tehran, \\ Iran
}

\section{*Correspondence to \\ Mona Zamanian Azodi, Proteomics Research Center (PRC), Darband St., Qods Sq., Tehran, Iran. \\ Tel: +982122714248 \\ Email: mona.azodi@gmail.com}

Published online December 1 2019

\begin{abstract}
Introduction: The human melanoma is a type of invasive tumor the treatment of which is challenging. To better understand the proton irradiation mechanisms as one of the widely applied therapy for this type of cancer, bioinformatics analysis of proteomics outcome could be beneficial. Methods: Protein-protein interaction network analysis of the differentially expressed proteins (DEPs) of melanoma BLM (BRO lung metastasis) cells in the treatment of 3 Gy dosage proton therapy was performed in this study via Cytoscape V.3.7.2. and its integrated plug-ins.

Results: Eighteen DEPs were searched for network constructions and limited numbers of query +neighbor proteins were found central. The hub-bottlenecks (i.e. central nodes) were GAPDH, ACTB, ALB, AKT1, TP53, and EGFR. The fist mentioned proteins were from DEPs. The enrichment analysis of these elements identified nitric-oxide synthase regulator activity and the positive regulation of the norepinephrine uptake that may be the key to the mechanisms of proton therapy. Conclusion: In conclusion, the identified central nodes (EGFR, TP53, ALB, AKT1, GAPDH, and ACTB) and the related biological terms are the critical affected genes and biological terms in the irradiated melanoma cells.

Keywords: Human melanoma, Radiation, Network analysis, Biological process
\end{abstract}

\section{Introduction}

The uveal melanoma is a form of melanoma for the melanocytes in the eyes. ${ }^{1}$ This fatal malignancy frequently migrates to liver and causes some problems. ${ }^{2}$ It is known as the fast spreading tumor in which the chance of apoptosis is very low. One of the first treatment options for this type of metastatic tumor is radiotherapy. ${ }^{3}$ Furthermore, a form of improved radiotherapy, i.e. irradiation by a proton beam, is a potential tumor treatment method in terms of appropriate targeting and dosage spreading. ${ }^{4}$ The clinical outcome of this approach implies that the survival rate of this treatment is very acceptable and the mechanism by which this method works is recognized as the reduction of ocular toxic materials. In addition, it is estimated that only $7 \%-10 \%$ of these patients treated with proton irradiation require eye removal. ${ }^{5}$ What is more, it should be noted that treating Uveal melanoma is one of the sensitive procedures due to its location in eyes. ${ }^{6}$ The mechanism behind the effectiveness of this procedure is by ceasing the migration of melanoma cells. ${ }^{7}$

Understanding the mechanisms by which radiotherapy impacts on the tumor efficiently could be better recognized by high throughput methods such as proteomics. ${ }^{4}$ Proteomics introduces biomarkers which are important in the underlying mechanism of treatment procedure. These proteins are differentially expressed in the subject receiving the treatment. Further information can be revealed by assessing these differentially expressed proteins (DEPs) by bioinformatics, in a way that a set of DEPs can be introduced for network construction. In a protein-protein interaction network analysis, proteins are screened for interaction properties. Those with the highest values of interaction could be more promising with regard to biomarker properties. ${ }^{8-10}$ Two key criteria are usually proposed for network topological evaluation, namely degree and betweenness centrality. Proteins with the highest amount of these parameters are regarded as the central proteins (hub-bottlenecks) of a constructed

Please cite this article as follows: Zadeh-Esmaeel MM, Shahrokh S, Zamanian Azodi M, Ahmadi N. The highlighted role of GAPDH and nitricoxide synthase regulator activity in proton beam irradiated melanoma BLM cells. / Lasers Med Sci. 2019;10(suppl 1):S68-S72. doi:10.15171/ jlms.2019.S13. 
network. These elements are vital for network strength and function. Alterations in their expression could cause differentiation in the system of interactions. ${ }^{11}$ Consequently, in this study, proteins with differential expression in the treatment with proton are investigated more in terms of interaction properties to introduce the potential ones.

\section{Methods}

In this study, the proteomic evaluation of the BLM cell line as a metastatic melanoma cell line (derived from the lung embedded in a nude mouse) treated with a proton beam ( 3 Gy dosage) and $60 \mathrm{keV}$ and then cultured for 28-35 days was conducted. 13 up-regulated and 4 down-regulated proteins were detected with the fold change above the threshold of $1.5 .{ }^{4}$ More precisely, 18 proteins were found at first; however, one of them, namely LMNB2, had the fold change lower than 1.5 . We investigated all the 18 proteins as a network of protein interaction systems. Cytoscape 3.7.2 (https://cytoscape.org/) constructed and analyzed a network of these candidates as two networks. ${ }^{12}$ The first network consisted of only the 18 proteins, while the second network also included the surrounding proteins. In addition, STRING database v. 1.5.0 (http://string-db. org) was the source for network construction. This source provided different parts including queries for the disease name, the compound, the protein name, and PubMed. A kappa score (edge weight for physical interactions; from 0 to 1 ) of 0.4 as a default cut-off was considered. ${ }^{13}$ Centrality analysis was based on two common parameters, namely degree $(\mathrm{K})$ and betweenness centrality (BC). Proteins with high degree values are considered as hubs. Similarly, proteins with high values of $\mathrm{BC}$ are known as bottlenecks. The proteins with high values of both $\mathrm{K}$ and $\mathrm{BC}$ are called hub-bottlenecks. Hub-bottlenecks are the central proteins of an interacting network. The Network Analyzer focuses on the centrality features of an interacting network. ${ }^{14}$ To better understand the hub-bottlenecks, functional analysis in terms of biological process detection is carried out by ClueGO v.2.5.5. ${ }^{15}$ This application analyzes gene ontology (biological process, molecular functions, and cell component) and pathway enrichment. The statistical criteria for this analysis are as follows: the number of genes per term: 1 and the percentage of genes per term: 2 . Other considered criteria are: 1 . the usage of term fusions and 2 . showing only the statistically significant terms. Moreover, $P$ value was corrected with Bonferroni step down.

\section{Results}

Eighteen DEPs were introduced in the Cytoscape and an interaction pattern of them was achieved without any node addition via the STRING database (see Figure 1).

The next step is to add neighbor proteins to understand the full interaction properties of proteins in a whole interacting system. Fifty proteins were added to the DEPs and a network with 2 central properties is visualized

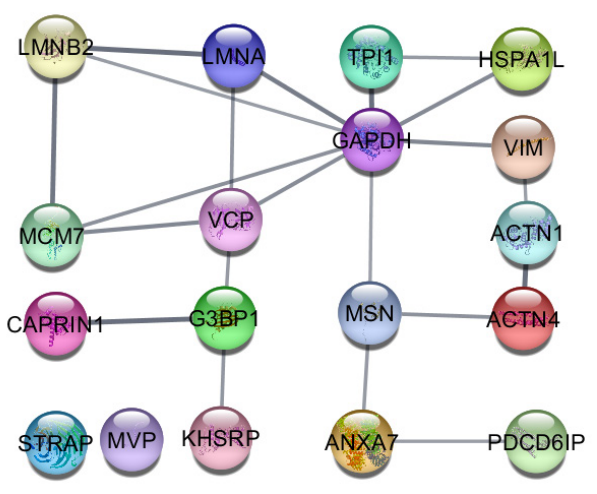

Figure 1. A Pattern of Interaction Among 18 Differentially Expressed Proteins (the confidence cut-off score: 0.4 , the number of additional nodes: 0 )

\section{in Figure 2.}

In this analysis from white to orange, the betweenness values change from lowest to highest. Likewise, the bigger the node sizes, the higher the degree amounts in Figure 2.

The values of centrality parameters are listed in Table 1 for more clarifications. Proteins are ranked based on the degree value. In this procedure, $10 \%$ of the nodes with the highest degree values and betweenness centrality were designated individually and the common nodes were assigned as the hub-bottlenecks of the network.

Six hub-bottlenecks were obtained from this analysis and the highest value of degree is 55 .

The enrichment analysis of hub-bottlenecks produced more information related to these elements. Six proteins were assessed by ClueGO for the identification of biological processes (see Figure 3).

In Figure 3, nitric-oxide synthase regulator activity and the positive regulation of norepinephrine uptake are the leading groups associated with the hub-bottlenecks. The group $P$ values were corrected with Bonferroni step down

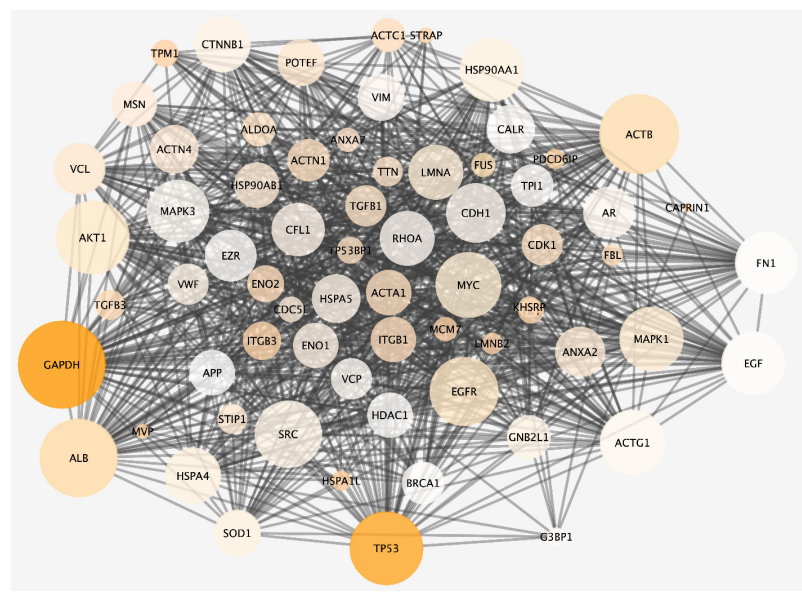

Figure 2. The Centrality Analysis of the Network of the DEPs Plus the Neighbors $(18+50$ nodes). Bigger node size refers high amount of degree value. Color changes (from white to yellow) denote to increase betweenness centrality. 
Table 1. The List of Hub-Bottlenecks of the Protein Interaction Network of Melanoma Cells Treated With Proton Radiation

\begin{tabular}{lcc}
\hline Display Name & Degree & Betweenness Centrality \\
\hline GAPDH $^{*}$ & 55 & 0.06 \\
ACTB & 50 & 0.02 \\
ALB & 49 & 0.03 \\
AKT1 & 46 & 0.02 \\
TP53 & 46 & 0.05 \\
EGFR & 43 & 0.02 \\
\hline
\end{tabular}

Note: The asterisk assigned node is from the query proteins.

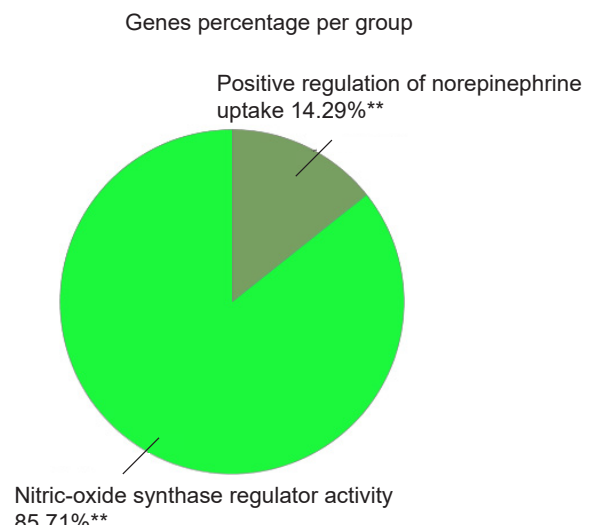

Figure 3. A pie chart view of two clusters of biological processes which are related to the hub-bottlenecks. The asterisk sign indicates the statistically significant group. The criteria such as min number of genes per term, the percentage of genes per term, and the Kappa cut-off score were considered as 1,2 , and $=0.5$ respectively.

for the first and second groups as 3.8E-08 and $6.6 \mathrm{E}-4$ respectively.

\section{Discussion}

Many investigations about therapeutic methods are affected by proteomics technology. Cancer diagnosis and treatment are the important fields which are of great concern to proteomics Proton treatment is an advanced approach for tumor managements. To decipher the underlying mechanisms of this method, it is essential to analyze the molecular basis of the treated samples. Proteomics is a promising study which investigates DEPs in a condition of treatment. Here, a set of proteins from a proteomics study were chosen for more analysis to get a better knowledge of their role in the mechanism of proton therapy. Protein-protein interaction network analysis as a bioinformatics approach provides the understanding of the interaction pattern of the DEPs. ${ }^{16-18}$ Those proteins with higher values of centrality in a network could be more promising as a contributor in the mechanism of proton irradiation action on melanoma cells. For this aim, as mentioned above, 18 proteins were chosen and a network of their interactions was constructed in two patterns. In the first network, no additional neighbor protein was added and this visualization demonstrated that the DEPs were in interactions as a component except two which remained as individual nodes. This result also refers to the importance of interaction concept that dysregulated proteins could influence their nearby proteins. In other words, neighbor proteins are affected by one dysregulated protein. The addition of 50 neighbor proteins and the analysis of their centrality indicated that there are some proteins with exceptional centrality properties. These proteins are called hub-bottlenecks, any dysregulation of which could result in a huge alteration in network topology. ${ }^{19}$

All of the central proteins (EGFR, TP53, ALB, AKT1, GAPDH, and ACTB) contribute to the nitric-oxide synthase regulator activity. The later one (ACTB) also participates in the positive regulation of norepinephrine uptake term.

Since among all the queried proteins, only GAPDH is a central protein, it is highlighted as a critical DEP in this study. GAPDH is the DEP exposed to the proton irradiation. GAPDH is a housekeeping protein; there are several documents about the alteration of its level in the body associated with different diseases. ${ }^{20-22}$ It has been reported that the deregulation of GAPDH happens in various types of cancers such as melanoma, lung cancer, prostatic cancer, colorectal cancer, renal cancer, pancreatic cancer, breast cancer, glioma, bladder cancer, gastric cancer, and liver cancer. In almost all cancers, GAPDH is up-regulated to provide a suitable growth condition for tumor cells. ${ }^{23}$

Dong et al reported that there is an inverse correlation between the increment of nitric oxide synthase activity and the occurrence of Metastasis in K-1735 cells of murine melanoma. ${ }^{24}$ Another investigation revealed that IL8 is regulated by nitric oxide in melanoma cells. ${ }^{25}$ An imbalanced relationship between nitric oxide and superoxide anion which is resulted from uncoupled nitric oxide synthase and affects human melanoma development is reported by Gonçalves et al. ${ }^{26}$ The positive regulation of norepinephrine uptake is another term that is attributed to the central nodes. There is evidence that stress hormones can promote the growth of B16-F10 melanoma metastases. ${ }^{27}$ It seems that the central nodes and the related biological terms are involved in the nature of the melanoma cell line in comparison with the radiation effect. It can be useful to consider the supplement treatment protocol associated with the radiotherapy.

\section{Conclusion}

The findings indicate that EGFR, TP53, ALB, AKT1, GAPDH, and ACTB as central proteins are related to nitric-oxide synthase regulator activity and the positive regulation of norepinephrine uptake; these two biological terms have contra-effects on metastasis promotion. Therefore, it can be concluded that these terms are 
attributed to the radiation effect and nature of melanoma cancer.

\section{Ethical Considerations}

Not applicable.

\section{Conflict of Interests}

The authors declare no conflict of interest.

\section{Acknowledgment}

Shahid Beheshti University of Medical Sciences supports this research.

\section{References}

1. Shain AH, Bagger MM, Yu R, Chang D, Liu S, Vemula S, et al. The genetic evolution of metastatic uveal melanoma. Nat Genet. 2019;51(7):1123-30. doi: 10.1038/s41588-0190440-9.

2. Angi M, Kalirai H, Prendergast S, Simpson D, Hammond $\mathrm{DE}$, Madigan MC, et al. In-depth proteomic profiling of the uveal melanoma secretome. Oncotarget. 2016;7(31):4962335. doi: 10.18632/oncotarget.10418.

3. Wang F, Bing Z, Zhang Y, Ao B, Zhang S, Ye C, et al. Quantitative proteomic analysis for radiation-induced cell cycle suspension in 92-1 melanoma cell line. J Radiat Res. 2013;54(4):649-62. doi: 10.1093/jrr/rrt010.

4. Kedracka-Krok S, Jankowska U, Elas M, Sowa U, Swakon J, Cierniak A, et al. Proteomic analysis of proton beam irradiated human melanoma cells. PLoS One. 2014;9(1):e84621. doi: 10.1371/journal.pone.0084621.

5. Verma V, Mehta MP. Clinical Outcomes of Proton Radiotherapy for Uveal Melanoma. Clin Oncol (R Coll Radiol). 2016;28(8):e17-e27. doi: 10.1016/j. clon.2016.01.034.

6. Levin W, Kooy H, Loeffler J, DeLaney T. Proton beam therapy. Br J Cancer. 2005;93(8):849-54. doi: 10.1038/ sj.bjc. 6602754 .

7. Jasińska-Konior K, Pochylczuk K, Czajka E, Michalik M, Romanowska-Dixon B, Swakoń J, et al. Proton beam irradiation inhibits the migration of melanoma cells. PloS One. 2017;12(10):e0186002. doi: 10.1371/journal. pone. 0186002

8. Rostami-Nejad M, Rezaei-Tavirani M, Zadeh-Esmaeel MM, Rezaei-Tavirani S, Akbari Z, Esmaeili S, et al Assessment of cytokine-mediated signaling pathway dysregulation in arm skin after $\mathrm{CO} 2$ laser therapy. $J$ Lasers Med Sci. 2019;10(4):257-63. doi: 10.22037/jlms. v10i4.26591.

9. Rezaei-Tavirani M, Mansouri V, Tavirani MR, RostamiNejad M, Bashash D, Azodi MZ. Gene and Biochemical Pathway Evaluation of Burns Injury via Protein-Protein Interaction Network Analysis. Galen Medical Journal. 2019;8:1257. doi: 10.31661/gmj.v8i0.1257.

10. Rezaei-Tavirani M, Rezaei Tavirani M, Akbari Z, Hajimehdipoor H. Prediction of coffee effects in rats with healthy and NAFLD conditions based on proteinprotein interaction network analysis. Research Journal of Pharmacognosy. 2019;6(4):7-15. doi: 10.22127/ rjp.2019.93500.
11. Rezaei Tavirani M, Rezaei Tavirani S, Zadeh-Esmaeel MM, Ahmadi NA. Introducing Critical Pain-related Genes: A System Biology Approach. Basic and Clinical Neuroscience. 2019;10(4):401-8. doi: 10.32598/bcn.9.10.310.

12. Smoot ME, Ono K, Ruscheinski J, Wang PL, Ideker T. Cytoscape 2.8: new features for data integration and network visualization. Bioinformatics. 2011;27(3):431-2. doi: 10.1093/bioinformatics/btq675.

13. Szklarczyk D, Gable AL, Lyon D, Junge A, Wyder S, HuertaCepas J, et al. STRING v11: protein-protein association networks with increased coverage, supporting functional discovery in genome-wide experimental datasets. Nucleic Acids Res. 2019;47(D1):D607-D613. doi: 10.1093/nar/ gky1131.

14. Assenov Y, Ramírez F, Schelhorn SE, Lengauer T, Albrecht M. Computing topological parameters of biological networks. Bioinformatics. 2008;24(2):282-4. doi: 10.1093/ bioinformatics/btm554.

15. Bindea G, Mlecnik B, Hackl H, Charoentong P, Tosolini M, Kirilovsky A, et al. ClueGO: a Cytoscape plug-in to decipher functionally grouped gene ontology and pathway annotation networks. Bioinformatics. 2009;25(8):1091-3. doi: 10.1093/bioinformatics/btp101.

16. Safari-Alighiarloo N, Taghizadeh M, Rezaei-Tavirani M, Goliaei B, Peyvandi AA. Protein-protein interaction networks (PPI) and complex diseases. Gastroenterol Hepatol Bed Bench. 2014;7(1):17-31. doi: 10.22037/ghfbb. v7i1.511.

17. Rezaei-Tavirani M, Rezaei-Tavirani S, Mansouri V, Rostami-Nejad M, Rezaei-Tavirani M. Protein-protein interaction network analysis for a biomarker panel related to human esophageal adenocarcinoma. Asian Pac J Cancer Prev. 2017;18(12):3357-63. doi: 10.22034/ APJCP.2017.18.12.3357.

18. Rezaei-Tavirani M, Rezaei-Tavirani S, Ahmadi N, Naderi $\mathrm{N}$, Abdi S. Pancreatic adenocarcinoma protein-protein interaction network analysis. Gastroenterol Hepatol Bed Bench. 2017;10(1):S85-S92. doi: 10.22037/ghfbb.v0i0.1265.

19. Tavirani MR, Mansouri V, Tavirani SR, Tackallou $\mathrm{SH}$, Rostami-Nejad M. Gliosarcoma protein-protein interaction network analysis and gene ontology. Int J Cancer Manag. 2018;11(5):e65701.doi: 10.5812/ijcm.65701.

20. Barber RD, Harmer DW, Coleman RA, Clark BJ. GAPDH as a housekeeping gene: analysis of GAPDH mRNA expression in a panel of 72 human tissues. Physiol Genomics. 2005;21(3):389-95. doi: 10.1152/ physiolgenomics.00025.2005.

21. Delunardo F, Soldati D, Bellisario V, Berry A, Camerini $S$, Crescenzi $M$, et al. Anti-GAPDH autoantibodies as a pathogenic determinant and potential biomarker of neuropsychiatric diseases. Arthritis Rheumatol. 2016;68(11):2708-16. doi: 10.1002/art.39750.

22. Tristan C, Shahani N, Sedlak TW, Sawa A. The diverse functions of GAPDH: views from different subcellular compartments. Cell Signal. 2011;23(2):317-23. doi: 10.1016/j.cellsig.2010.08.003.

23. Guo C, Liu S, Sun MZ. Novel insight into the role of GAPDH playing in tumor. Clin Transl Oncol. 2013;15(3):167-72. doi: 10.1007/s12094-012-0924-x.

24. Dong Z, Staroselsky AH, Qi X, Xie K, Fidler IJ. Inverse 
correlation between expression of inducible nitric oxide synthase activity and production of metastasis in K-1735 murine melanoma cells. Cancer Res. 1994;54(3):789-93.

25. Andrew P, Harant H, Lindley I. Nitric oxide regulates IL-8 expression in melanoma cells at the transcriptional level. Biochem Biophys Res Commun. 1995;214(3):949-56. doi: 10.1006/bbrc.1995.2378.

26. Gonçalves DA, Xisto R, Gonçalves JD, da Silva DB, Soares JPM, Icimoto MY, et al. Imbalance between nitric oxide and superoxide anion induced by uncoupled nitric oxide synthase contributes to human melanoma development. Int J Biochem Cell Biol. 2019;115:105592. doi: 10.1016/j. biocel.2019.105592.

27. Valles SL, Benlloch M, Rodriguez ML, Mena S, Pellicer JA, Asensi M, et al. Stress hormones promote growth of B16-F10 melanoma metastases: an interleukin 6-and glutathionedependent mechanism. J Transl Med. 2013;11(1):72. doi: 10.1186/1479-5876-11-72. 